\title{
An explicit construction of quantum codes from one-generator generalized quasi-cyclic codes
}

\author{
Yu Yao, Yuena $\mathrm{Ma}^{*}$, Husheng Li, and Jingjie Lv \\ Air Force Engineering University, Department of Basic Sciences, 710051Xi'an, P. R. China
}

\begin{abstract}
In this paper, we take advantage of a class of one-generator generalized quasi-cyclic (GQC) codes of index 2 to construct quantum errorcorrecting codes. By studying the form of Hermitian dual codes and their algebraic structure, we propose a sufficient condition for self-orthogonality of GQC codes with Hermitian inner product. By comparison, the quantum codes we constructed have better parameters than known codes.
\end{abstract}

\section{Introduction}

Quantum error-correcting codes (QECCs) have significant application value in quantum communication and quantum computing. Shor proposed the concept of QECCs in 1995 [1]. After the connection between classical error correction codes and QECCs was established, more and more researchers began to be interested in this aspect of work. With the development of research, the work has been extended to the $q$-ary QECCs. Many QECCs with good parameters have been constructed [2-5].

Generalized quasi-cyclic (GQC) codes are the remarkable generalization of cyclic codes and quasi-cyclic (QC) codes. Moreover, compared to QC codes, GQC codes have arbitrary length that made GQC codes are an important class of linear codes. The concept of GQC codes was proposed by Siap and Kulhan in 2005 [6]. Many high-performance LDPC codes are not QC codes but GQC codes [7-9]. Therefore, many researchers considered its structures and got many good results $[10,11]$.

Thus, we can use GQC codes to construct QECCs. Galindo et al. [12] first constructed QECCs via two-generator QC codes. Afterwards Lv et al. utilized the two-generator QC codes [13] and one-generator QC $[14,15]$ codes to construct QECCs, respectively, and gave many codes with good parameters. Inspired by these works, we consider one-generator GQC codes to construct QECCs. To our knowledge, few researches in this area involve.

The paper is organized as follows. Section 2 summarizes some preliminary concepts and results. In Section 3, we provide a construction of one-generator GQC codes which are Hermitian self-orthogonal. Section 4 utilizes the class of GQC codes to construct QECCs and gives examples of QECCs with good parameters. The paper is summarized with a discussion in Section 5.

\footnotetext{
**Corresponding author: xakgd2020@163.com
} 


\section{Preliminaries}

In this paper, let $C$ be a code of length $n$, dimension $k$ and minimum Hamming distance $d$ over the finite field $\mathbb{F}_{q^{2}}^{n}$, where $\frac{k}{n}$ is called information rate. For any two vectors $u=\left(u_{0}, u_{1}, \cdots, u_{n-1}\right), \quad v=\left(v_{0}, v_{1}, \cdots, v_{n-1}\right) \in \mathbb{F}_{q^{2}}^{n}$, their Hermitian inner product is defined as $\langle u, v\rangle_{H}=\sum_{j=0}^{n-1} u_{j}^{q} v_{j}$, where $\alpha^{q}=\bar{\alpha}$ denote its conjugation. the Hermitian dual code of $C$ is defined as $C^{\perp_{H}}=\left\{v \in \mathbb{F}_{q^{2}}^{n} \mid\langle u, v\rangle_{H}=0, \forall u \in C\right\}$.

Let $C$ be a $q^{2}$-ary linear code of length $n_{1}+n_{2}$. For any codeword $c=\left(c_{0}, c_{1}, \cdots, c_{n_{1}-1}, c_{n_{1}}, c_{n_{1}+1}, \cdots, c_{n_{1}+n_{2}-1}\right) \in C \quad, \quad$ if $\quad \varphi(c)=\left(c_{n_{1}-1}, c_{0}, \cdots, c_{n_{1}-2}, c_{n_{1}+n_{2}-1}, c_{n}, \cdots\right.$, $\left.c_{n_{1}+n_{2}-2}\right) \in C$, then the code $C$ is called a GQC code of length $n_{1}+n_{2}$ and index $l=2$ over $\mathbb{F}_{q^{2}}$. Note that, when $n_{1}=n_{2}, C$ is a QC code. Furthermore if $l=1$, then $C$ is a cyclic code.

According to the following theorem, we can know the dimension of a GQC code, which depends on its parity-check polynomial.

Theorem 1 [11] Let $h(x)$ be the parity-check polynomial of a 1-generator GQC code $C$ of block lengths $\left(m_{1}, m_{2}, \cdots, m_{l}\right)$ with generator $\left(a_{1}(x), a_{2}(x), \cdots, a_{l}(x)\right)$. Then,

$a-h(x)=\operatorname{lcm}_{1 \leq i \leq l}\left\{\frac{x^{m_{i}}-1}{g_{i}(x)}\right\}$, where $g_{i}(x)=\operatorname{gcd}\left(a_{i}(x), x^{m_{i}}-1\right)$.

$b$ - as a vector space over $\mathbb{F}_{q}$, dimension of $C$ is $\operatorname{deg}(h(x))$.

A $q$-ary QECC of length $n$ and dimension $k$ is represented by $\llbracket n, k, d \rrbracket_{q}$, where $d$ denotes the minimum distance and means that this code can detect $d-1$ errors and correct $\left[\frac{d-1}{2}\right]$ errors. From the following theorem, we can construct QECCs by classical Hermitian self-orthogonal linear codes.

Theorem 2 [2] If a Hermitian self-orthogonal $[n, k]_{q^{2}}$ linear code $C$ exists, then a $\llbracket n, n-2 k, d \rrbracket_{q}$ QECC yields, where no vectors of weight less than $d$ in $C^{\perp_{H}} \backslash C$.

\section{Hermitian self-orthogonal GQC codes}

Next, a class of self-orthogonal one-generator GQC codes with the Hermitian inner product will be defined to construct QECCs.

Definition 1 If $\operatorname{gcd}\left(q, n_{1}\right)=\operatorname{gcd}\left(q, n_{2}\right)=1$, let $C$ be a GQC code over $\mathbb{F}_{q^{2}}$ of length $n_{1}+n_{2}$ and index 2 generated by $\left(g_{1}(x), f(x) g_{2}(x)\right)$, where $g_{1}(x)\left|x^{n_{1}}-1, g_{2}(x)\right| x^{n_{2}}-1$ and $\operatorname{gcd}\left(f(x),\left(x^{n_{2}}-1 / g_{2}(x)\right)\right)=1$.

Throughout this paper, we set $g(x)=g_{0}+g_{1} x+\cdots+g_{n-1} x^{n-1}$, and define the polynomials $g^{q}(x)=g_{0}^{q}+g_{1}^{q} x+\cdots+g_{n-1}^{q} x^{n-1}$. Moreover, when $g(x) h(x)=x^{n}-1$, then 
$g^{\perp}(x)=x^{\operatorname{deg} h(x)} h\left(\frac{1}{x}\right)$. Besides, $[g(x)]$ denotes the vector, which decided by the polynomial $g(x)$ coefficients over $\mathbb{F}_{q^{2}}^{n}$.

Next, the algebraic structure of the Hermitian dual code of $C$ will be given and a sufficient condition for self-orthogonality with Hermitian inner product will be proposed.

Theorem 3 If a GQC code $C$ satisfies $\operatorname{gcd}\left(\frac{g_{2}(x)}{g_{1}(x)}, x^{n_{1}}-1\right)=1, g_{1}^{\perp q}(x)\left|g_{1}(x)\right| g_{2}(x)$, $g_{2}^{\perp q}(x) \mid g_{2}(x)$ and $x^{n_{1}}-1 \mid x^{n_{2}}-1$, Then there exist

(1) $C^{\perp_{H}}$ is generated by $\left(g_{1}^{\perp q}(x), f(x) g_{2}^{\perp q}(x)\right),\left(g_{1}(x), 0\right)$ and $\left(g_{1}^{\perp q}(x), 0\right)$, where $C^{\perp_{H}}$ is the Hermitian dual code of $C$ over $\mathbb{F}_{q^{2}}$.

(2) $C$ is Hermitian self-orthogonal.

Proof (1) we first prove that $C^{\perp_{H}}$ is generated by $\left(g_{1}^{\perp q}(x), f(x) g_{2}^{\perp q}(x)\right),\left(g_{1}(x), 0\right)$ and $\left(g_{1}^{\perp q}(x), 0\right)$.

I. Let $c_{1}=\left(\left[a(x) g_{1}(x)\right],\left[a(x) f(x) g_{2}(x)\right]\right)$ denote any codeword in $C$, and $c_{2}=\left(\left[b(x) g_{1}^{\perp q}(x)+c(x) g_{1}(x)+d(x) g_{1}^{\perp q}(x)\right],\left[b(x) f(x) g_{2}^{\perp q}(x)\right]\right) \quad$ represent any codeword in the code $D$, which generated by $\left(g_{1}^{\perp q}(x), f(x) g_{2}^{\perp q}(x)\right),\left(g_{1}(x), 0\right)$ and $\left(g_{1}^{\perp q}(x), 0\right)$, where $a(x), b(x), c(x)$ and $d(x)$ are arbitrary polynomials over $\mathbb{F}_{q^{2}}[x]$. Then $\left\langle c_{1}, c_{2}\right\rangle_{H}=\left\langle\left[a(x) g_{1}(x)\right],\left[(b(x)+d(x)) g_{1}^{\perp q}(x)\right]\right\rangle_{H}+\left\langle\left[a(x) g_{1}(x)\right],\left[c(x) g_{1}\right]\right\rangle_{H}+\left\langle\left[a(x) f(x) g_{2}(x)\right]\right.$ , $\left.\left[b(x) f(x) g_{2}^{\perp q}(x)\right]\right\rangle_{H}$. Because $\left\langle g^{\perp q}(x)\right\rangle$ is Hermitian dual code of cyclic code $\langle g(x)\rangle$, and for arbitrary polynomials $k(x)$ over $\mathbb{F}_{q^{2}}[x],\langle k(x) g(x)\rangle$ is the subcode of $\langle g(x)\rangle$. Obviously, $\left\langle c_{1}, c_{2}\right\rangle_{H}=\left\langle\left[a(x) g_{1}(x)\right],\left[c(x) g_{1}\right]\right\rangle_{H}=0 \quad$ when $\quad g_{1}^{\perp q}(x) \mid g_{1}(x)$. Thus, the Hermitian dual code $C^{\perp_{H}}$ includes the code $D$.

II. Moreover, according to Theorem 1, the dimension of $C$ is $\operatorname{dim}(C)=$ $\operatorname{deg}\left(\operatorname{lcm}\left\{\frac{x^{n_{1}}-1}{g_{1}(x)}, \frac{x^{n_{2}}-1}{g_{2}(x)}\right\}\right) \cdot x^{n_{1}}-1\left|x^{n_{2}}-1, g_{1}(x)\right| g_{2}(x)$ and $\operatorname{gcd}\left(\frac{g_{2}(x)}{g_{1}(x)}, x^{n_{1}}-1\right)=1$, thus $\frac{x^{n_{1}}-1}{g_{1}(x)} \mid \frac{x^{n_{2}}-1}{g_{2}(x)}$. and then $\operatorname{dim}(C)=n_{2}-\operatorname{deg} g_{2}(x)$. On the other hand, due to $g_{1}^{\perp q}(x) \mid g_{1}(x)$ and $g_{2}^{\perp q}(x) \mid g_{2}(x), \operatorname{deg}\left(\operatorname{lcm}\left\{\frac{x^{n_{1}}-1}{g_{1}^{\perp q}(x)}, \frac{x^{n_{2}}-1}{g_{2}^{\perp q}(x)}\right\}\right)=\operatorname{deg} \frac{x^{n_{2}}-1}{g_{2}^{\perp q}(x)}=\operatorname{deg} g_{2}(x)$. It is easy to know that $\operatorname{dim}(D)=\operatorname{deg} g_{2}(x)+\left(n_{1}-\operatorname{deg} g_{1}(x)\right)+\operatorname{deg} g_{1}(x)=n_{1}+\operatorname{deg} g_{2}(x)$, which is exactly equal to the dimension of $C^{\perp_{H}}$. So $C^{\perp_{H}}$ is generated by $\left(g_{1}^{\perp q}(x), f(x) g_{2}^{\perp q}(x)\right)$, $\left(g_{1}(x), 0\right)$ and $\left(g_{1}^{\perp q}(x), 0\right)$.

(2) Next we prove that $C$ is Hermitian self-orthogonal. It is directly derived from the following equation:

$$
\left(g_{1}(x), f(x) g_{2}(x)\right)=\frac{g_{2}(x)}{g_{2}^{\perp q}(x)}\left(g_{1}^{\perp q}(x), f(x) g_{2}^{\perp q}(x)\right)+\left(g_{1}(x), 0\right)-\frac{g_{2}(x)}{g_{2}^{\perp q}(x)}\left(g_{1}^{\perp q}(x), 0\right) .
$$


So, when $g_{2}^{\perp q}(x) \mid g_{2}(x), C \subseteq C^{\perp_{H}}$.

In summary, our conclusion is proved.

\section{Construction of QECCs}

We can straightforward draw the following theorem via Theorem 2 and 3.

Theorem 4 Let $\operatorname{gcd}\left(\frac{g_{2}(x)}{g_{1}(x)}, x^{n_{1}}-1\right)=1, \quad g_{1}^{\perp q}(x)\left|g_{1}(x)\right| g_{2}(x), \quad g_{2}^{\perp q}(x) \mid g_{2}(x) \quad$ and $x^{n_{1}}-1 \mid x^{n_{2}}-1$. Then there exist Hermitian self-orthogonal code $C$ and QECC with parameters $\llbracket n_{1}+n_{2}, n_{1}+2 \operatorname{deg} g_{2}(x)-n_{2}, d_{\min }\left(C^{\perp_{H}}\right) \|_{q}, \quad$ where $d_{\text {min }}\left(C^{\perp_{H}}\right)$ denotes the distance of Hermitian dual code $C^{\perp_{H}}$.

Next, we compare the new QECCs with existing ones to illustrate that many QECCs with good parameters can be constructed from Hermitian self-orthogonal GQC codes.

Example 1. Assume that $q=4, n_{1}=7$ and $n_{2}=21$. Let $\omega$ be a primitive element of $\quad \mathbb{F}_{16} \quad$ Let $\quad g_{1}(x)=x^{4}+x^{3}+x^{2}+1 \quad$ and $g_{2}(x)=x^{15}+x^{14}+\omega^{5} x^{13}+\omega^{5} x^{12}+x^{11}+\omega^{10} x^{10}+x^{9}$ $+\omega^{5} x^{8}+x^{7}+x^{6}+x^{5}+\omega^{10} x^{4}+\omega^{5} x^{3}+\omega^{10} x^{2}+\omega^{10} x+1$. Set $f(x)=\omega x^{2}+x+1$ then a GQC code $C$ with parameters $[28,6,12]_{16}$ obtained, which satisfies Theorem 4 . Naturally, we obtain a $\llbracket 28,16,3 \rrbracket_{4}$ quantum code with information rate 0.571 . Furthermore, it has better parameters than $\llbracket 26,14,3 \rrbracket_{4}$ with information rate 0.538 in [16].

Example 2. Now suppose that $q=4, n_{1}=15$ and $n_{2}=45$. Set $\omega$ be a primitive element of $\mathbb{F}_{16}$. Take the $g_{1}(x)=x^{13}+\omega^{2} x^{12}+\omega^{6} x^{11}+\omega^{6} x^{10}+\omega^{13} x^{9}+\omega^{14} x^{8}+\omega^{8} x^{7}+$ $\omega^{14} x^{6}+\omega^{2} x^{5}+\omega^{13} x^{4}+\omega^{3} x^{3}+x^{2}+\omega^{8} x+\omega^{3} \quad, \quad g_{2}(x)=x^{40}+\omega^{2} x^{39}+\omega^{6} x^{38}+x^{37}+\omega^{6} x^{36}$ $+\omega^{9} x^{35}+\omega^{3} x^{34}+\omega^{9} x^{33}+\omega^{12} x^{32}+\omega^{12} x^{31}+\omega^{4} x^{30}+\omega^{5} x^{29}+\omega^{28}+\omega^{6} x^{27}+\omega^{3} x^{26}+\omega^{3} x^{25}$ $+\omega^{10} x^{24}+\omega^{11} x^{23}+\omega^{11} x^{22}+\omega^{3} x^{21}+\omega^{4} x^{20}+\omega^{12} x^{19}+\omega^{7} x^{18}+\omega^{9} x^{16}+\omega^{11} x^{15}+x^{14}+\omega^{11} x^{13}$ $+\omega x^{12}+\omega^{13} x^{11}+\omega^{7} x^{10}+\omega^{13} x^{9}+\omega x^{8}+\omega^{9} x^{7}+\omega^{4} x^{6}+\omega^{11} x^{4}+\omega^{13} x^{3}+\omega^{2} x^{2}+\omega^{10} x+\omega^{5}$. And select $f(x)=\omega^{3} x^{4}+x^{3}+x^{2}+x+1$. We know $C$ is a GQC code with parameters $[60,5,15]_{16}$. By Theorem 4, a $\llbracket 60,50,3 \rrbracket_{4}$ quantum code with information rate 0.833 is obtained. In comparison with the $\llbracket 65,53,3 \rrbracket_{4}$ quantum code with information rate 0.815 in [16], it has better parameters.

\section{Conclusion}

In this paper, we consider a class of one-generator GQC codes with index 2 and study the form of its Hermitian dual code. Moreover, a sufficient condition to decide the Hermitian self-orthogonality of GQC codes is provided, so that many QECCs with good parameters can be constructed by these GQC codes effectively. Therefore, our method achieves both feasible and practical values.

This work is supported by National Natural Science Foundation of China (Nos.11801564, 11901579). 


\section{References}

1. P. W. Shor, Phys. Rev. A, Gen. Phys. 52(4), 2493-2496 (1995)

2. A. Ashikhmin, E. Knill, IEEE Trans. Inf. Theory 47(7), 3065-3072 (2001)

3. D. Gottesman, Ph.D. Thesis, California Institute of Technology (1977)

4. A. Ketkar, A. Klappenecker, S. Kumar, IEEE Trans. Inf. Theory 52, 4892-4914 (2006)

5. S. Ling, J.Luo, C. Xing, IEEE Trans. Inf. Theory 56, 4080-4084 (2008)

6. I. Siap, N. Kulhan, Appl. Math. 5, 24-30 (2005)

7. Y. Kou, S. Lin, M. P. C. Fossorier, IEEE Trans. Inf. Theory 47(7), 2711-2736 (2001)

8. H. Tang, J. Xu, S. Lin, K. A. S. Abdel-Ghaffar, IEEE Trans. Inf. Theory 51(2), 572-596 (2005)

9. V.Y. Van, H. Matsui, S. Mita,: IEEE Global Telecommunications Conference GLOBECOM, Miami, FL, USA 1-6 (2010)

10. Y. Cao, Codes Cryptogr. 60, 67-79 (2011)

11. M. Esmaeili, S. Yari, AAECC. 20, 159-173 (2009)

12. C. Galindo, F. Hernando, R. Mastsumoto, Finite Fields Appl. 52, 261-280 (2018)

13. J. Lv, R. Li, J. Wang, IEEE Communications Letters, 1-1 (2020)

14. J. Lv, R. Li, J.Wang, International Journal of Theoretical Physics. 59, 300-312 (2020)

15. J. Lv, R. Li, J.Wang, IEEE Access 7, 85782-85785 (2019)

16. Y. Edel, Table of quantum twisted codes. electronic address: www.mathi.uniheidelberg.de/yves/matritzen/QTBCH/QTBCHIndex.html 